\title{
Network Structures of Internet Gaming Disorder and Gaming Disorder: Symptom Operationalization Causes Variation
}

Matúš Adamkovič 1,2,3, Marcel Martončik ${ }^{1,2,3}$, Veli-Matti Karhulahti ${ }^{4}$, Ivan Ropovik ${ }^{4,5}$

${ }^{1}$ Faculty of Arts, University of Presov, Slovakia

2 Institute of Social Sciences CSPS SAS, Slovakia

${ }^{3}$ Department of Music, Art and Culture Studies, Faculty of Humanities and Social Sciences, University of Jyväskylä, Finland

${ }^{4}$ Institute for Research and Development of Education, Faculty of Education, Charles University, Czechia

${ }^{5}$ Faculty of Education, University of Presov, Slovakia

\section{ORCID}

Matúš Adamkovič: 0000-0002-9648-9108

Marcel Martončik: 0000-0003-4869-6900

Veli-Matti Karhulahti: 0000-0003-3709-5341

Ivan Ropovik: 0000-0001-5222-1233

\section{Authors' Note}

Preprint version 3.0, 07.10.2022. MA, MM, and VMK are joint first authors of this paper. We have no known conflict of interests to disclose.

Correspondence concerning this article should be addressed to Marcel Martončik, Institute of Social Sciences CSPS SAS, Karpatská 1337/5, 040 01, Slovakia. Email: martoncik@ protonmail.ch 


\section{Abstract}

Background: From 2022, the ICD-11 includes the first mental disorder based on digital technology, "gaming disorder", which was previously suggested as a condition for further study in the DSM-5 (2013). In this cross-sectional study, we provide the first large-scale network analysis of various symptom structures for these constructs to understand the complex interconnections between their proposed symptoms.

Methods: Culturally diverse samples of 2,846 digital game players ( $M=25.3$ years) and 746 esports players ( $M=23.5$ years) were recruited. A network approach was applied to explore a multiverse of gaming disorder symptom structures, effects of item operationalization, and possible external moderators. Gaming disorder was measured using the IGDS9-SF, GDT and several items borrowed from C-IGDS, PIE-9, and C-VAT 2.0 scales.

Results: Two symptoms (loss of control and continued use despite problems) present in both, the DSM-5 and ICD-11, were systematically central to most of the analyzed networks. Alternative operationalizations of single items systematically caused significant network differences. Networks were invariant across groups of play style, age, gender, gaming time, and most of the psychosocial characteristics.

Discussion: Our results caution practitioners and researchers when studying and interpreting gaming disorder symptoms. The data indicate that even minor operational changes in symptoms can lead to significant network-level changes, thus highlighting the need for careful wording.

Keywords: gaming disorder; internet gaming disorder; network analysis; network approach; operationalization 
As billions of people now play digital games globally, gaming has come to serve as a highly important leisure activity for children, adolescents, and adults. At the same time, the first mental disorder based on digital technology, "gaming disorder" (GD), has entered global clinical use in 2022 and a current meta-analysis estimates $2.4 \%$ of people to develop pathological gaming patterns (Kim et al., 2021). This diagnostic category refers to both offline and online digital game play under the addictive behaviors class in the World Health Organization's ICD-11 (WHO, 2018). So far, the American Psychiatric Association has not included digital play behaviors in the DSM-5 (APA, 2013), however, "internet gaming disorder" (IGD) is listed as a potential condition needing more research.

The current knowledge regarding IGD/GD symptoms ${ }^{1}$ is limited. The nine symptoms proposed in the DSM-5 (see Table 1) were soon found weak in utility (e.g., Griffiths et al., 2016); and while the ICD-11 criteria tend to be considered more useful, new clinical evidence implies that the majority of treatment-seekers do not meet these criteria either (Starcevic et al., 2020).

Table 1

Description of IGD/GD symptoms and criteria

Internet gaming disorder (DSM-5)

Persistent and recurrent use of the Internet to engage in games, often with other players, leading to clinically significant impairment or distress as indicated by five (or more) of the following in a 12month period:

Symptoms

\section{Preoccupation}

Preoccupation with internet games. (The individual thinks about previous gaming activity or anticipates playing the next game; internet gaming becomes the dominant activity in daily life.)

\section{Withdrawal}

Withdrawal symptoms when internet gaming is taken away. (These symptoms are typically described as irritability, anxiety, or sadness, but there are no physical signs of pharmacological withdrawal.)

\section{Tolerance}

Tolerance-the need to spend increasing amounts of time engaged in internet games.

\section{Loss of control}

Unsuccessful attempts to control the participation in internet games.

\section{Loss of interests}

Loss of interests in previous hobbies and

entertainment as a result of, and

with the exception of, internet games.

\section{6 . Continued use}

\section{Gaming disorder (ICD 11)}

Persistent or recurrent gaming behavior ('digital gaming' or 'video-gaming'), which may be online (i.e., over the internet) or offline, manifested by:

\section{Criteria}

\section{Loss of control}

Impaired control over gaming (e.g., onset, frequency, intensity, duration, termination, context).

\section{Prioritization}

Increasing priority given to gaming to the extent that gaming takes precedence over other life interests and daily activities.

\section{3a. Continued use \\ Continuation or escalation of gaming despite the occurrence of negative consequences. 3b. Problems \\ The behavior pattern is of sufficient severity to result in significant impairment in personal, family, social, educational, occupational or other important areas of functioning.}

1 The DSM-5 uses the term "symptom" and the ICD-11 "criterion" for their listed diagnostic requirements. We use the former due to its consistency with the network approach, however, we also note our skepticism toward the causal implication of symptoms being produced by a latent construct (IGD/GD). 
Continued excessive use of internet games despite knowledge of psychosocial problems.

\section{Deception}

Has deceived family members, therapists, or others regarding the amount of internet gaming.

\section{Escape}

Use of Internet games to escape or relieve a

negative mood (e.g., feelings of helplessness, guilt,

anxiety).

\section{Problems}

Has jeopardized or lost a significant relationship, job, or education or career opportunity because of

participation in internet games.

Little is known about the relationships between the proposed symptoms. Our study responds to this need from the perspective of network analysis (see, e.g., Borsboom et al., 2021), which is well suited for exploring the structures of relationships between symptoms - those which have and have not been included in diagnostic manuals (Fried et al., 2016; Hansen et al., 2021).

\section{Network analysis}

To account for the complexity of psychological phenomena, network analysis has become widely applied in the field of psychopathology (Borsboom \& Cramer, 2013; Borsboom, 2021; Borsboom et al., 2021). In network analysis, symptoms of a disorder are assumed to act as independent causal agents, mutually affecting each other. Under this approach, psychopathology is modeled as a complex system, emerging from self-sustaining recurrent interactions of causally linked symptoms. Network analysis then allows one to explore complex patterns of symptom relationships and identify which symptoms are central and which are peripheral to the given disorder (see Borsboom et al., 2021). The approach helps effectively communicate the relationships in complex data-statistically taking into consideration not only selected item relationships but those of the entire network. Specifically, a network visualizes how the symptoms (represented as nodes) are connected (links between the nodes), conditional on all other symptoms in the network. If there is no connection between two symptoms, it means that the pair is conditionally independent given the other symptoms in the network. On the other hand, if two symptoms are conditionally dependent, the connection is represented by a line between the two. Unlike in correlation analysis, the connection of two symptoms may change whenever other symptom-level changes occur in the network.

\section{Present study}

In this exploratory study, our goal was to study the linkages between the different network structures of gaming disorder, based on DSM-5 and ICD-11 symptomatologies, and different operationalizations of symptoms varying in content validity. Previously, some symptoms of IGD/GD have been labeled as "core" and other as "peripheral," however, the literature is mixed regarding which should belong to each class (e.g., Ballou \& van Rooij, 2021; Snodgrass et al., 2018). The present study was designed to pursue clarity on this issue as well. We formulated research questions 1.1. and 1.2. before data collection, and they additionally led us to ask 1.3, 1.4, and 1.5 during the analyses: 
RQ 1.1 How are the IGD/GD symptoms structurally interconnected?

RQ 1.2 What are the central and peripheral symptoms in the IGD/GD networks?

RQ 1.3 How is the (non-overlapping) combination of IGD/GD symptoms structurally interconnected?

RQ 1.4 Which symptoms bridge the IGD and GD constructs?

RQ 1.5 How does the inclusion of causally relevant variables from outside of the description of IGD/GD in the diagnostic manuals-craving, neglect of own physical health, and gaming timechange the combined IGD/GD network?

The preliminary state of GD research is also mirrored by the large number of screening scales, the items of which collectively measure hundreds of differently operationalized symptoms (King et al., 2020). In theory, after the DSM-5 (2013) and ICD-11 (2019) were published, new screening scales should have a shared ontology. However, content validity analysis shows that scales following the diagnostic manuals operationalize their symptoms in substantially different ways - thus measuring potentially different constructs (Karhulahti et al., 2021). Whether these different operationalizations result in different screening outcomes remains an open empirical question, and our network approach is a fitting method for seeking answers to it. We choose to investigate the operationalization of four DSM-5 symptoms that previous research (ibid.) found weak in content validity across scales. We formulated 2.1, 2.2, 2.3, and 2.4 before data collection, and they led us to ask 2.5 during analysis:

How do different operationalizations of "withdrawal" (RQ 2.1), "loss of interests" (RQ 2.2), "tolerance" (RQ 2.3), and "continued use" (RQ 2.4) affect the DSM-5 network?

RQ 2.5 How is the combination of DSM-5 network with different operationalizations of "withdrawal", "loss of interests", "tolerance", and "continued use" together with causally relevant variables from outside of the diagnostic manuals-craving, neglect of own physical health, and gaming time-structurally interconnected?

As Wittgenstein (1953) pointed out already in the 1950s, "games" represent such a rich conceptual category that one such item (e.g., olympic games) may have almost nothing to do with another (e.g., children's games of roleplay). This issue, known as family resemblance, is a serious challenge for IGD/GD measurement too, as different groups of people play different games that potentially lack a common denominator. For instance, many digital games designed for children are different from those for adults, and the recently emerged competitively oriented play, esports-now having hundreds of millions of players globally-represents a distinct type of gaming (Karhulahti, 2020). The above issues should be further examined within different player groups to better understand if and how symptoms are similarly interconnected across varying player populations. All research questions in the third group were formulated before data collection.

How do the IGD and GD networks differ between groups of play style (RQ 3.1), age (RQ 3.2), gender (RQ 3.3), gaming time (RQ 3.4) and psychosocial characteristics (RQ 3.5)?

\section{Methods}

\section{Participants}

Data from an international, culturally diverse sample $(N=3015)$ of digital game players was collected via the Prolific platform (for all details, see supplementary materials). Several screening 
procedures aimed at data quality were carried out (bot detection, failed attention checks, and careless responding patterns). As a result, $5.6 \%$ of the participants were excluded from analyses. The final sample size was $\mathrm{N}=2846$, consisting of digital game players ( $80.5 \%$ male, $18.2 \%$ female, $1 \%$ non-binary) with a mean age of 25.3 years (SD $=7.4)$. The average reported time spent gaming equaled 3.97 hours per day $(S D=2.48)(61.11 \%$ of this time was dedicated to online gaming, on average). The participants spent another 3.06 hours per day $(S D=2.88)$ on additional activities related to digital games (watching gaming-related videos, etc.). For RQ 3.3, a similar sample of esports-specific players ( $N=801$, after screening for careless participants, $6.9 \%$ of the sample was excluded, resulting in the effective sample of $\mathrm{N}=746$; see supplementary materials) was recruited. Given the exploratory nature of this study, we acquired a maximally large sample (as defined by the resources provided by our funder) and verified the stability of the networks via post hoc analysis.

\section{Measures}

DSM-5-based IGD was measured using the English version of Internet Gaming Disorder Scale Short-Form (IGDS9-SF; Pontes \& Griffiths, 2015). ICD-11 based GD was measured using the English version of the Gaming Disorder Test (Pontes et al., 2019). For RQ2, we selected alternative symptom operationalizations with high content validity, based on a previous semantic analysis (Karhulahti et al., 2021). Items measuring withdrawal, loss of interests, and continued use were selected from C-IGDS (Sigerson et al., 2017); tolerance from PIE-9 (Pearcy et al., 2016). For RQ 3.4, we also measured moderator variables that reflected psychosocial characteristics (support within family, motives for gaming, problematic gaming cognitions, self-control, neuroticism, harm avoidance, loneliness, and reward responsiveness). For RQs 1.5 and 2.5, we measured craving, neglect of one's own physical health, and gaming time which have been suggested and found to be relevant non-diagnostic variables (Castro-Calvo et al., 2021; van Rooij et al., 2017; APA, 2013). Operationalizations of the symptoms, their endorsement, and descriptive statistics are presented in Table 2 and Table 3.

\section{Table 2}

Operationalization of IGD/GD symptoms and their endorsement

\begin{tabular}{|c|c|c|c|}
\hline \multirow[t]{2}{*}{ Symptom } & \multirow[t]{2}{*}{ Operationalization } & \multicolumn{2}{|c|}{$\begin{array}{l}\text { Percentage of participants } \\
\text { endorsing the item } \\
\text { (responded 'often' or 'very } \\
\text { often') }\end{array}$} \\
\hline & & $\begin{array}{l}\text { regular } \\
\text { gamers }(N= \\
2846)\end{array}$ & $\begin{array}{l}\text { eports } \\
\text { gamers }(N= \\
743)\end{array}$ \\
\hline & IGDS9-SF (Pontes \& Griffiths, 2015) & & \\
\hline preoccupation & $\begin{array}{l}\text { Do you feel preoccupied with your gaming } \\
\text { behaviour? (Some examples: Do you think } \\
\text { about previous gaming activity or } \\
\text { anticipate the next } \\
\text { gaming session? Do you think gaming has } \\
\text { become the dominant activity in your daily } \\
\text { life?) }\end{array}$ & 16.23 & 22.34 \\
\hline withdrawal & $\begin{array}{l}\text { Do you feel more irritability, anxiety or } \\
\text { even sadness when you try to either reduce }\end{array}$ & 4.78 & 8.21 \\
\hline
\end{tabular}




\begin{tabular}{|c|c|c|c|}
\hline \multirow[b]{2}{*}{ tolerance } & \multicolumn{3}{|l|}{ or stop your gaming activity? } \\
\hline & $\begin{array}{l}\text { Do you feel the need to spend increasing } \\
\text { amount of time engaged gaming in order to } \\
\text { achieve satisfaction or pleasure? }\end{array}$ & 9.52 & 14.80 \\
\hline loss of control & $\begin{array}{l}\text { Do you systematically fail when trying to } \\
\text { control or cease your gaming activity? }\end{array}$ & 6.47 & 10.36 \\
\hline loss of interests & $\begin{array}{l}\text { Have you lost interests in previous hobbies } \\
\text { and other entertainment activities as a } \\
\text { result of your engagement with the game? }\end{array}$ & 9.55 & 13.86 \\
\hline continued use & $\begin{array}{l}\text { Have you continued your gaming activity } \\
\text { despite knowing it was causing problems } \\
\text { between you and other people? }\end{array}$ & 8.50 & 14.27 \\
\hline deception & $\begin{array}{l}\text { Have you deceived any of your family } \\
\text { members, therapists or others because the } \\
\text { amount of your gaming activity? }\end{array}$ & 2.85 & 4.85 \\
\hline escape & $\begin{array}{l}\text { Do you play in order to temporarily escape } \\
\text { or relieve a negative mood (e.g., } \\
\text { helplessness, guilt, anxiety)? }\end{array}$ & 35.73 & 41.32 \\
\hline \multirow[t]{3}{*}{ problems } & $\begin{array}{l}\text { Have you jeopardised or lost an important } \\
\text { relationship, job or an educational or } \\
\text { career opportunity because of your gaming } \\
\text { activity? }\end{array}$ & 2.85 & 5.52 \\
\hline & At-risk participants (total score $>=36$ ) & 0.63 & 1.47 \\
\hline & GDT (Pontes et al., 2019) & & \\
\hline loss of control & $\begin{array}{l}\text { I have had difficulties controlling my } \\
\text { gaming activity. }\end{array}$ & 9.28 & 11.98 \\
\hline prioritization & $\begin{array}{l}\text { I have given increasing priority to gaming } \\
\text { over other life interests and daily activities. }\end{array}$ & 14.65 & 22.75 \\
\hline continued use & $\begin{array}{l}\text { I have continued gaming despite the } \\
\text { occurrence of negative consequences. }\end{array}$ & 15.11 & 19.38 \\
\hline \multirow[t]{2}{*}{ problems } & $\begin{array}{l}\text { I have experienced significant problems in } \\
\text { life (e.g., personal, family, social,education, } \\
\text { occupational) due to the severity of my } \\
\text { gaming behavior. }\end{array}$ & 4.29 & 7.13 \\
\hline & $\begin{array}{l}\text { At-risk participants (at least } 4 \text { points on all } \\
4 \text { symptoms) }\end{array}$ & 1.26 & 3.77 \\
\hline \multicolumn{4}{|c|}{$\begin{array}{l}\text { High content validity operationalizations of selected symptoms } \\
\text { Note: Based on (Karhulahti et al., 2021) these items proved higher content } \\
\text { validity than original items in IGDS9-SF }\end{array}$} \\
\hline & C-IGDS (Sigerson et al., 2017) & & \\
\hline $\begin{array}{l}\text { withdrawal } \\
\text { symptoms (A) }\end{array}$ & $\begin{array}{l}\text { Do you feel irritable, anxious, or sad when } \\
\text { Internet gaming is taken away? }\end{array}$ & 7.55 & 10.77 \\
\hline $\begin{array}{l}\text { loss of interests } \\
\text { (A) }\end{array}$ & $\begin{array}{l}\text { Have you experienced loss of interests in } \\
\text { previous hobbies and entertainment as a } \\
\text { result of, and with the exceptions of, } \\
\text { Internet games? }\end{array}$ & 10.08 & 14.67 \\
\hline $\begin{array}{l}\text { continued } \\
\text { excessive use }(A)\end{array}$ & $\begin{array}{l}\text { Do you continue to use Internet games } \\
\text { excessively despite knowledge of }\end{array}$ & 18.55 & 24.90 \\
\hline
\end{tabular}




\begin{tabular}{|c|c|c|c|}
\hline \multicolumn{4}{|c|}{ psychosocial problems? } \\
\hline \multirow[b]{2}{*}{ tolerance $(A)$} & PIE-9 (Pearcy et al., 2016) & & \\
\hline & $\begin{array}{l}\text { Do you find an increasing need to spend } \\
\text { increasing amounts of time engaged in } \\
\text { digital games? }\end{array}$ & 8.04 & 12.92 \\
\hline \multicolumn{4}{|c|}{ Possible additional symptoms } \\
\hline & C-VAT 2.0 (van Rooij et al., 2017) & & \\
\hline craving & $\begin{array}{l}\text { How often have you had a strong urge } \\
\text { (desire) to play digital games? }\end{array}$ & 28.67 & 33.92 \\
\hline $\begin{array}{l}\text { neglect of one's } \\
\text { own physical } \\
\text { health }\end{array}$ & $\begin{array}{l}\text { How often have you neglected your own } \\
\text { health because of gaming? (examples: not } \\
\text { getting enough sleep, showering less, } \\
\text { failing to brush teeth, drinking } \\
\text { insufficiently). }\end{array}$ & 16.44 & 23.69 \\
\hline
\end{tabular}

Note: (A) - alternative operationalization.

Table 3

Descriptive statistics

\begin{tabular}{lccc}
\hline & regular players & esports players & $\begin{array}{c}\text { possible } \\
\text { range }\end{array}$ \\
\cline { 2 - 3 } gaming time & $M(S D)$ & $M(S D)$ & \\
IGDS9-SF & $3.97(2.48)$ & $5.37(3.00)$ & $0-24$ \\
GDT & $2.03(0.62)$ & $2.33(0.66)$ & $1-5$ \\
craving & $2.08(0.81)$ & $2.32(0.84)$ & $1-4$ \\
neglect of own physical health & $3.05(0.93)$ & $3.21(0.99)$ & $1-5$ \\
family relationship & $2.36(1.13)$ & $2.62(1.19)$ & $1-5$ \\
social motive for gaming & $2.25(0.50)$ & $2.23(0.51)$ & $1-3$ \\
competition motive for gaming & $2.48(0.83)$ & $2.75(0.78)$ & $1-5$ \\
escape motive for gaming & $2.86(0.99)$ & $3.33(0.89)$ & $1-5$ \\
coping motive for gaming & $3.14(1.05)$ & $3.19(1.03)$ & $1-5$ \\
gaming cognition & $3.03(0.76)$ & $3.04(0.75)$ & $1-5$ \\
neuroticism & $1.53(0.42)$ & $1.65(0.46)$ & $1-3$ \\
harm avoidance & $2.83(0.75)$ & $2.90(0.70)$ & $1-5$ \\
loneliness & $3.41(0.65)$ & $3.28(0.65)$ & $1-5$ \\
self-control & $3.13(0.77)$ & $3.17(0.76)$ & $1-5$ \\
reward responsiveness & $2.97(0.64)$ & $2.87(0.61)$ & $1-5$ \\
\hline
\end{tabular}

Additional information about participants, data collection, and measures can be found at https://osf.io/pdym4.

\section{Statistical analysis}

Networks modeling the nine IGD as well as the four GD symptoms were estimated using the EBICglasso method. The centrality/connectivity measures as well as edge-weights were obtained, and the nodes were compared in their strength. Networks reflecting four different operationalizations of IGD symptoms were estimated and the network comparison test (van Borkulo et al., 2017) was used to assess differences in the parameters (centrality measures and edge/weights) between the IGD structure based on a standardized measure and alternative 
operationalizations. To examine the effects of each operationalization in more detail, the networks in which only one item was substituted at the time were estimated and compared. The network comparison test was also used to examine whether the network structures are invariant across different levels of moderating variables. The continuous moderators were dichotomized using the conditional inference trees method (Jones et al., 2020); the optimal threshold was determined by iteratively testing a particular network structure across all levels of the moderating variable up until the largest difference (when applicable) in the networks of the respective subgroups was found. The differences in network structure, global strength, and the proportion of significantly different edges and nodes were extracted. The analyses were performed in $\mathrm{R}$, with bootnet (Epskamp et al., 2018), NetworkComparisonTest (van Borkulo et al., 2017), and networktree (Jones et al., 2020). For more technical details, see supplementary materials.

\section{Results}

RQ1. The visualizations of the network structures of IGD/GD and the corresponding centrality plots are displayed in Figure 1. The symptoms of withdrawal, tolerance, loss of control, and continued use appear as central symptoms in the IGD network. For GD, loss of control played the most central role.

Figure 1

Visualization of IGD and GD networks and their centrality/connectivity indices

A

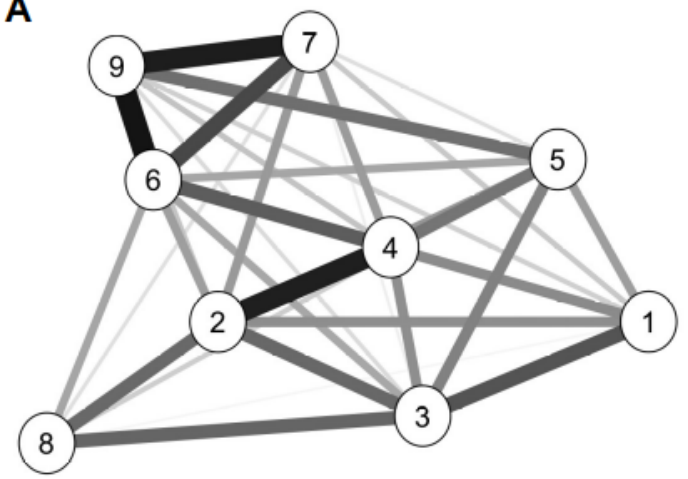

C

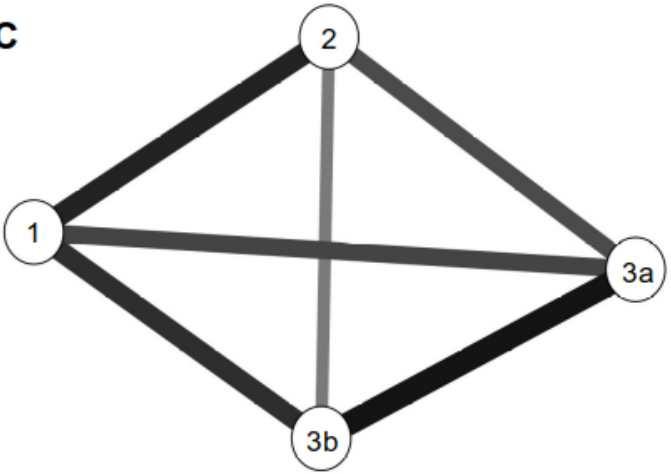

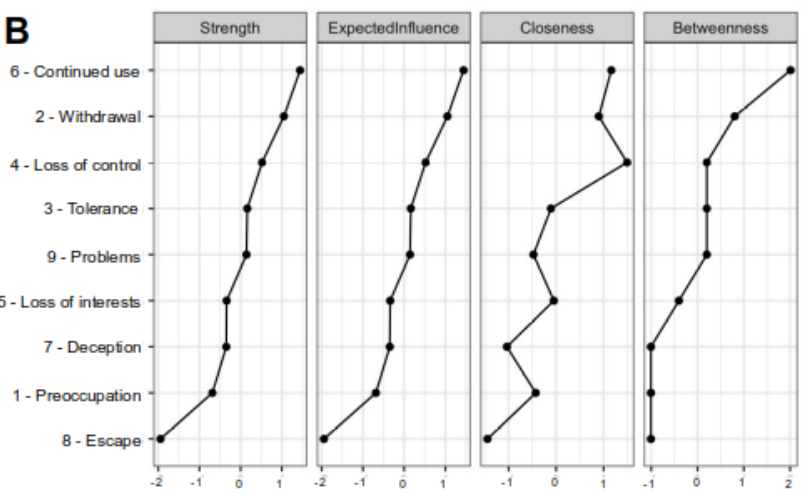

。

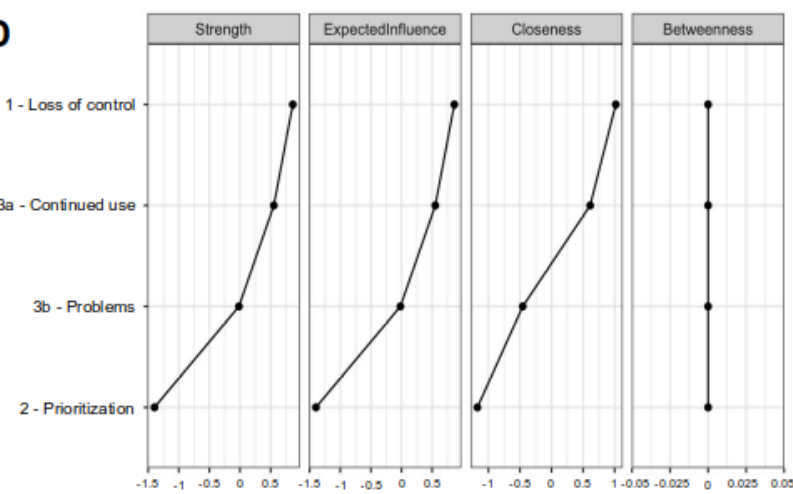

Note: $\mathrm{A}=$ visualization of the IGD network; $\mathrm{B}=$ centrality/connectivity indices for the IGD network; $C=$ visualization of the GD network; $D=$ centrality/connectivity indices for the GD network

For a visualization of the combined network see Figure 2. Prioritization symptom was the bridge indicator with the highest strength. In both networks, with operationalizations based on a standardized measure and alternative operationalizations including causally relevant variables 
from outside of the diagnostic manuals, craving was among the strong nodes, neglect of own physical health was among the weak nodes, and gaming time was the weakest of all nodes.

\section{Figure 2}

Visualization of a combined IGD/GD network and bridge symptoms strength

A

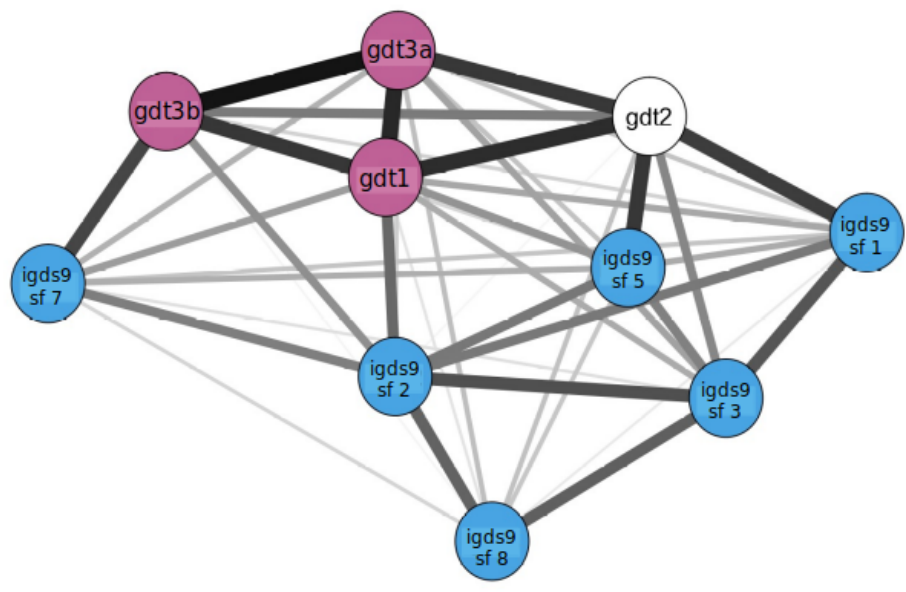

B

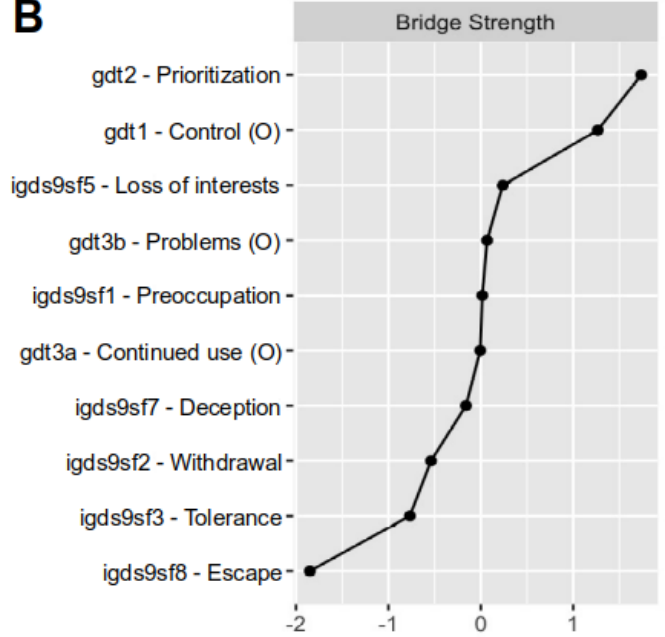

Note: $\mathrm{A}=$ visualization of the combined network (GD symptoms are in magenta, non-overlapping IGD symptoms are in blue; the strongest bridge symptom is in white); $\mathrm{B}=$ bridge symptoms strength

RQ2. The network structure of IGD (by IGDS9-SF) and that with four higher-content-validity operationalizations were non-invariant, but the global strength was invariant across the two structures (i.e., the network with the original operationalizations and the network with the alternative operationalizations). Out of the nine symptoms, a significant difference in centrality indices was observed for continued use (see Figure 3). We also created new networks for each of the four alternative operationalizations one-by-one: in two of the four networks, the change in a single item alone (withdrawal and continued use) caused a significant network-level change. 


\section{Figure 3}

Centrality/connectivity indices networks of IGD involving IGDS9-SF items and four alternative higher-content-validity operationalizations
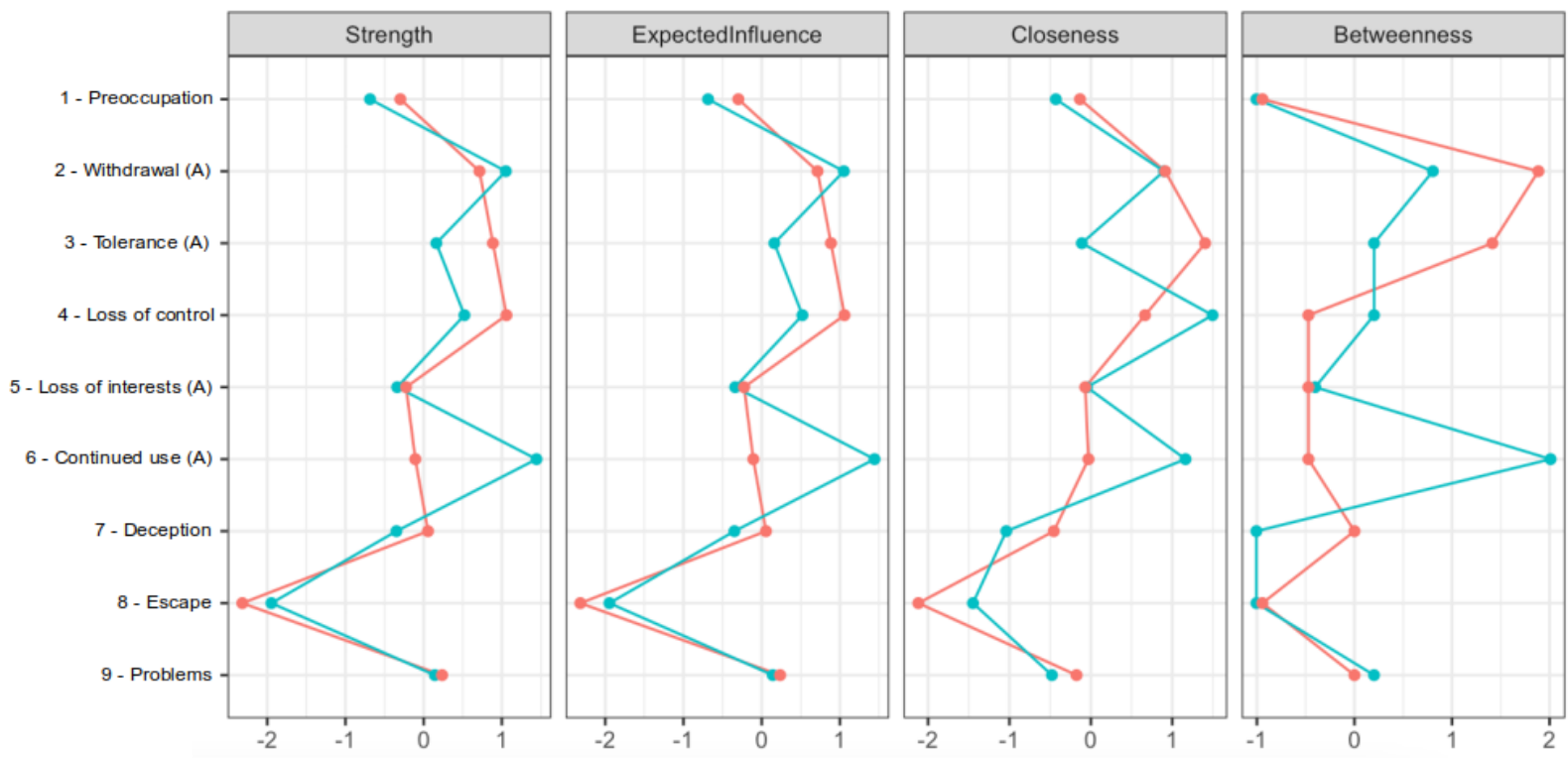

Note: Blue color represents parameters for the original IGDS9-SF items. Red color represents parameters for higher-content-validity operationalizations. For the red color, the alternative items are marked by (A). P-values for difference in strength in the pairs of items: $1=.087,2=.041,3$ $=.061,4=.352,5=.639,6=<.001,7=.270,8=.674,9=.978$.

RQ3. Both IGD and GD networks were invariant across play styles (general digital game players vs. esports players; $p s>.57$ ) even when re-tested only with those who self-identified as esports players. Neither age, gender, nor gaming time ( $p s>.23$ ) were significant moderators of IGD/GD networks. Testing for network invariance across levels of all 11 psychosocial variables revealed that the IGD network was invariant in terms of both structure and global strength across all of them, while the GD network structure was significantly moderated by social and escape motives ( $p s<.05$ ) for gaming and its global strength was non-invariant for self-control and neuroticism ( $p s<.02)$.

\section{Supplementary analyses and sensitivity analyses}

Elaborated outcomes, additional research questions, and replication of the analyses on a sample of esports players can be found at https://osf.io/pdym4. Sensitivity analyses are incorporated into the R code that can be found at https://osf.io/pe9zd/.

\section{Discussion}

Our main findings shed light on the structural importance of specific symptoms, their operationalizations in particular, and the absence of network differences between different groups of players. Importantly, when we refer to the "importance" or "influence" of symptoms in 
this discussion, we explicitly refer to the statistical parameters. This does not necessarily imply clinical or theoretical importance and influence (unless specified otherwise).

\section{RQ 1 Symptoms in the networks}

In all DSM-5 based IGD networks, escape was systematically the weakest and least influential symptom. Conceptually, this is consistent with the previous findings where gaming for mood management also occurs in non-problematic play (Lemmens et al., 2015; Rehbein et al., 2015). Although recent literature (Stenseng et al., 2021) has suggested different operationalizations to reflect different kinds of escape and only those involving "self-suppression" to be IGD-relevant, in our data escape was weak and non-influential despite self-suppressive operationalization-thus yielding counterevidence for the above. The prevalence of escape in our data (see Table 2) was extremely high, which implies that, at least by the current operationalization, the self-reporting of this symptom also includes non-pathological escapism patterns. Notably, the ICD-11 does not list any mood management as a criterion for GD.

Second, we found loss of control to be the strongest and most influential symptom in both IGD and GD networks. On the other hand, the second ICD-11 symptom, prioritization, was found to be a weak and the least influential symptom within the network. This corroborates the view of 29 international experts who rated the symptom as the least relevant of all four ICD-11 symptoms (Castro-Calvo et al., 2021). This supports the idea (Kuss et al., 2017) that prioritizing gaming in daily life is rather common for healthy people who play digital games. Of note, while prioritization may serve as a (non-sufficient necessary) symptom, as listed in ICD-11, considering that many DSM-5 based instruments include it as one of the nine symptoms in polythetic measurement, scholars should remain skeptical toward such models.

One symptom that has not been included in the diagnostic manuals, craving, was among the strongest nodes in both networks. Craving was also extremely prevalent in both of our samples (almost a third of the respondents expressed craving "often" or "very often"), which suggests that, indeed, it is unlikely to serve as a "symptom" but rather precedes the problems, as evidenced by the high prevalence in the general population. One possible causal scenario is that (i) craving manifests as an outcome of an individual interacting with their culture and environment, and while it is not problematic for most individuals who successfully regulate their craving, for some with (ii) loss of control it may gradually begin to accumulate various life problems and ultimately (iii) continued use despite the problems, the latter two forming the core of the ICD-11 based network.

Two other non-listed symptoms, neglect of one's own physical health and time spent gaming, played only a minor role in all respective networks, which supports their lack of relevance for the current diagnostic constructs. Alternatively, these results could be explained by operationalization: for instance, the neglect of own's physical health was adjusted to include relatively common examples such as skipped teeth brushing and sleep hours, and as such, the symptom was highly prevalent in both samples (approximately a fifth of all participants). If the type and severity of these health problems would be readjusted, the relevance of this symptom could change as well. Moreover, the relevance of such non-official potential symptoms could also be different in the networks of clinical, treatment-seeking samples. In future research, it will be important to investigate whether the symptom networks of clinical and non-clinical participants differ. Such differences could yield valuable evidence regarding variables that may serve as preconditioning "risk factors" and variables that are actually caused by and symptomatic of a disordered network. 
Resulting hypothesis: Loss of control forms the essence of gaming-related problems by contributing to derivative life issues, some of which are currently listed as diagnostic symptoms (e.g., jeopardizing opportunities) while others are not (e.g., family conflicts). Future research should focus on better understanding "loss of control" as a construct and investigating how some people end up lacking control while others efficiently maintain control (see Smith 2022; Sripada 2021).

Our results are in line with some previous findings but in conflict with others. The two previous network analysis studies on IGD, Yuan et al. (2022) and Gomez et al. (2022) found loss of control and continued use to be strongest and most influential symptoms in the network; however, the strongest and most influential symptom in the former group's network, preoccupation, was of mediocre influence in our data. This could be due to variation in operationalizing preoccupation, as discussed below. Likewise, one of our three strong and influential IGD symptoms, withdrawal, has been considered lacking utility for diagnostic purposes by experts (e.g., Castro-Calvo et al., 2021) and was accordingly excluded from the most recent criteria list in the ICD-11. On the other hand, recent studies (e.g., Ballou \& Zendle, 2021) have indicated withdrawal to be among the potential signifiers of gaming-related health problems. Closely examining the present operationalization of withdrawal leads us to suggest the following: unlike in other network studies, the symptom is influential in our data because the IGDS9-SF item (see Table 2) does not measure withdrawal symptoms alone but also control issues ("when you try to either reduce or stop"). Therefore, what appears as the significance of withdrawal in our data actually corroborates the significance of loss of control. It remains for future studies to assess whether this reasoning is correct.

Based on our findings in the context of previous literature, there is a high probability that loss of control and continued use (despite related health problems) represent the most relevant symptoms of the ones that are currently listed in diagnostic manuals. Notably, these two symptoms were also the sole criteria for pathological gambling in the DSM III (APA, 1980), yet were later replaced by a large number of diverse symptoms for the use of polythetic measurement (DSM IV, DSM-5). Future analyses will hopefully help us better understand the nature of the construct and its relationship to diagnostic manuals (see Haslam 2003).

The role of other symptoms remains difficult to assess-to a large degree due to the numerous different ways in which they have been interpreted and operationalized (see below). Nonetheless, we found prioritization as the weakest symptom in the GD network, but as the strongest in the combined IGD/GD network. This implied a bridge function, which we tested and confirmed. The role of prioritization seems to be similar to that of craving, as discussed earlier. We interpret this as evidence for prioritization to be a potential peripheral symptom, albeit it remains to be seen how it should be applied in clinical practice (e.g., it may not be useful with individuals in treatment). Resulting hypothesis: Prioritizing or craving gaming are not problematic or diagnostically symptomatic, however, if occurring with loss of control, they may together lead to continued use and subsequent life issues. Future research should focus on better understanding continued use despite problems as a construct and investigate how such use may link to life issues. Etiological studies, in turn, should look into the differences of prioritization and craving contexts to more accurately identify cases where they may lead to problems.

\section{RQ2. Operationalization}

One of the foremost challenges in the field is that both DSM-5 and ICD-11 based IGD/GD screening instruments operationalize the official diagnostic criteria in many different ways 
(Karhulahti et al., 2021). This means that results produced by one instrument can differ significantly from those of another instrument-even when both claim to measure the same symptoms. Our findings are the first to provide strong evidence regarding this question: changes in only four item operationalizations already alter the dynamics of the entire network. Updating IGD items continued use, withdrawal, tolerance, and loss of interests into items with arguably higher content validity (of identically defined symptoms) resulted in network changes in the first three cases. Continued use (despite negative consequences), which was the strongest symptom in IGDS9-SF ("Have you continued your gaming activity despite knowing it was causing problems between you and other people?"), became weaker after re-operationalization ("Do you continue to use digital games excessively despite knowledge of psychosocial problems?", adapted CIGDS). Similarly, withdrawal symptoms ("Do you feel more irritability, anxiety or even sadness when you try to either reduce or stop your gaming activity?", IGDS9-SF) lost strength and network influence when updated ("Do you feel irritable, anxious, or sad when gaming is taken away?", adapted CIGDS). On the other hand, tolerance, which was originally a weak symptom ("Do you feel the need to spend an increasing amount of time engaged in gaming in order to achieve satisfaction or pleasure?", IGDS9-SF), became stronger after revision ("Do you find an increasing need to spend increasing amounts of time engaged in digital games?", adapted PIE-9).

Although our data and methods do not allow making inferences about which operationalizations are "better" or "correct" (or if any of them are), the findings are so far perhaps the most disconcerting evidence suggesting researchers to exercise caution when comparing survey results produced by different IGD/GD items and instruments. As we see, item-level changes can lead to significant network-level changes. Previous content validity studies have suggested that rating scales may only be interchangeable indicators if their item content overlaps (Fried, 2017) - our present findings provide direct empirical support for this. The next steps in the field should be to start assessing the validity of actively applied screening instruments and their items with clinically, culturally, and otherwise diverse populations, which can eventually lead to an understanding of how to make useful real-world inferences of various statistical cutoffs and outcomes.

Resulting hypothesis: Future replications of the present study, which apply measurement instruments of different content validity, will produce network models that differ from ours by symptom influence and relevance. In other words, our results cannot be reproduced by using instruments with different content validity.

\section{RQ3. Group differences}

We were unable to detect any meaningful group differences based on play style, age, gender, gaming time, and psychosocial characteristics in both networks. The networks and centrality measures were very similar in all groups, suggesting an absence of different mechanisms between symptoms across the subgroups. Especially interesting is the absence of significant differences between esports and other digital game players. We interpret this as evidence for esports games/gaming to operate in a relatively similar manner with other digital games and play, i.e., regardless of esports involvement, people do not differ in their DSM-5 and ICD-11 symptomatologies. It is possible that competitive and collaborative elements-which define esports gaming - are such common design components across digital game genres that statistical differences between players of genres are not meaningful. Because all of our participants were highly engaged in terms of daily play hours, future research should investigate whether null results 
can also be obtained between highly active players and those who play only occasionally (e.g., a few hours per week vs few hours per day).

Although our analyses were carried out with the symptoms suggested by the current diagnostic authorities, we cannot know if our measured variables are clinically relevant or if some clinically relevant (yet unknown) variables should have been included. With an improved future clinical understanding, exclusions and inclusions of variables can further improve network analyses. Finally, it is known that the symptomatologies of psychiatric constructs can differ between cultures (e.g., Kleinman, 1988), thus it remains important for future studies to investigate whether different cultural contexts might require different symptomatological descriptions. Taken together, our findings voice a need for collaborative efforts to pursue more coherent operationalization, symptomatology, and careful application of the present diagnostic manuals in both clinical practice and research.

Resulting hypothesis: Based on the systemic invariance between groups in our data, we expect that different demographic groups will not produce meaningfully different symptom networks. However, it remains important for future studies to explore possible network noninvariance in clinically and culturally different groups.

\section{Limitations and implications for future research}

We have identified several potential caveats of the present study which should be reflected upon. (1) The exploratory nature of the estimated networks requires future replication on both general as well as clinical (or at-risk) populations. Furthermore, by making our data open, we welcome other researchers to test for the robustness of the present findings using different analytical procedures. (2) The cross-sectional design of the study naturally limits the possibility of making evidence-based claims about causality of the processes. (3) Although the present study provides evidence for invariance of the networks across demographic and psychosocial characteristics, culturally specific factors underlying (disordered) gaming habits should be further studied. Related to this, we applied English instruments for measurement, which may have been difficult to interpret for some of our non-native English speaker respondents. (4) Moreover, as the re-operationalized items were not designed to operate in a network with the rest of the analyzed items, future studies should pursue comparative analyses between multiple complete IGD and GD instrument networks. (5) Finally, the data-driven approach we have utilized produced insights into the structure of disordered gaming and related variables and the importance of their operationalization. Nonetheless, future studies should aim to verify the verisimilitude of our conclusions and complement them with qualitative approaches, especially with actual treatmentseeking participants.

\section{Conclusions}

The findings of this study add to the accumulating knowledge on gaming-related health in three ways. By utilizing the symptomatology of current diagnostic manuals (IGD in DSM-5, GD in ICD-11) with samples of active digital game players $(n=2846)$ and esports players $(n=746)$, we applied network analysis and found loss of control as well as continued use despite problems the most central in the networks. Some symptoms, like escapism, were found to be clearly noninfluential. Better understanding of the symptoms as constructs and causal agents are needed to assess their clinical relevance. As an interesting counterpoint to the above, we found alternative operationalizations of individual symptoms to cause network-level changes of centrality and influence. In other words, the differences in how researchers choose to verbalize symptoms in 
their surveys seems to have an effect on symptoms relevance. This finding calls for collaborative efforts to coordinate and unify measurement. Finally, we found all networks invariant across multiple demographic groups and in esports/non-esports comparison. It seems unlikely that demographic variables significantly affect psychometric validity in gaming-related health measurement; however, future research should further investigate clinical and cultural differences-starting from the assessment of construct meaning and validity of each symptom.

\section{Data sharing}

Sharing the data was approved along with the permission to conduct the study by the Ethics committee of the Faculty of Arts, University of Presov, following the ethical principles stated in the Declaration of Helsinki. The data, $\mathrm{R}$ code, and ratings are openly available at https://osf.io/a8fhx/.

\section{Supplementary materials}

Additional information about participants, data collection, and measures can be found at https://osf.io/pdym4.

\section{Funding}

This work was supported by the Slovak Research and Development Agency under contracts no. APVV-18-0140, APVV-17-0418, and APVV-20-0319, the Scientific Grant Agency of the Ministry of Education, Science, Research and Sport of the Slovak Republic and Slovak Academy of Sciences (VEGA) under the contract no. 1/0217/20, Finnish Work Environment Fund (200349), and project PRIMUS/20/HUM/009. This project also received funding from the European Research Council (ERC) under the European Union's Horizon Europe research and innovation programme (grant agreement No 101042052).

\section{Authors' contributions}

MA: Conceptualization, Methodology, Formal analysis, Investigation, Data curation, Writing - Original Draft, Writing - Review \& Editing. MM: Conceptualization, Methodology, Investigation, Resources, Writing - Original Draft, Writing - Review \& Editing. VMK: Conceptualization, Methodology, Writing - Original Draft, Writing - Review \& Editing. IR: Conceptualization, Methodology, Formal analysis, Writing - Review \& Editing. First authorship shared by MA, MM, and VMK.

\section{References}

American Psychiatric Association. (2013). Diagnostic and statistical manual of mental disorders (5th ed.). Arlington, VA: American Psychiatric Publishing.

Ballou, N., \& Van Rooij, A. J. (2021). The relationship between mental well-being and dysregulated gaming: a specification curve analysis of core and peripheral criteria in five gaming disorder scales. Royal Society Open Science, 8(5), 201385. https://doi.org/10.1098/rsos.201385

Ballou, N., \& Zendle, D. (2022). "Clinically significant distress" in internet gaming disorder: An individual participant meta-analysis. Computers in Human Behavior, 129(107140), 107140. https://doi.org/10.1016/j.chb.2021.107140

Borsboom, D. (2017). A network theory of mental disorders. World Psychiatry, 16(1), 5-13. https://doi.org/10.1002/wps.20375 
Borsboom, D., \& Cramer, A. O. J. (2013). Network analysis: an integrative approach to the structure of psychopathology. Annual Review of Clinical Psychology, 9(1), 91-121. https://doi.org/10.1146/annurev-clinpsy-050212-185608

Borsboom, D., Deserno, M. K., Rhemtulla, M., Epskamp, S., Fried, E. I., McNally, R. J., Robinaugh, D. J., Perugini, M., Dalege, J., Costantini, G., Isvoranu, A.-M., Wysocki, A. C., van Borkulo, C. D., van Bork, R., \& Waldorp, L. J. (2021). Network analysis of multivariate data in psychological science. Nature Reviews Methods Primers, 1(1). https://doi.org/10.1038/s43586-021-00055-w

Castro-Calvo, J., King, D. L., Stein, D. J., Brand, M., Carmi, L., Chamberlain, S. R., Demetrovics, Z., Fineberg, N. A., Rumpf, H.-J., Yücel, M., Achab, S., Ambekar, A., Bahar, N., Blaszczynski, A., Bowden-Jones, H., Carbonell, X., Chan, E. M. L., Ko, C.-H., de Timary, P., ... Billieux, J. (2021). Expert appraisal of criteria for assessing gaming disorder: an international Delphi study. Addiction (Abingdon, England), 116(9), 2463-2475.

https://doi.org/10.1111/add.15411

Epskamp, S., Borsboom, D., \& Fried, E. I. (2018). Estimating psychological networks and their accuracy: A tutorial paper. Behavior Research Methods, 50(1), 195-212. https://doi.org/10.3758/s13428-017-0862-1

Fried, E. I. (2017). The 52 symptoms of major depression: Lack of content overlap among seven common depression scales. Journal of Affective Disorders, 208, 191-197. https://doi.org/10.1016/j.jad.2016.10.019

Fried, E. I., Epskamp, S., Nesse, R. M., Tuerlinckx, F., \& Borsboom, D. (2016). What are "good" depression symptoms? Comparing the centrality of DSM and non-DSM symptoms of depression in a network analysis. Journal of Affective Disorders, 189, 314-320. https://doi.org/10.1016/j.jad.2015.09.005

Gomez, R., Stavropoulos, V., Tullett-Prado, D., Schivinski, B., \& Chen, W. (2022). Network analyses of internet gaming disorder symptoms and their links with different types of motivation. BMC Psychiatry, 22(1), 76. https://doi.org/10.1186/s12888-022-03708-6

Griffiths, M. D., van Rooij, A. J., Kardefelt-Winther, D., Starcevic, V., Király, O., Pallesen, S., Müller, K., Dreier, M., Carras, M., Prause, N., King, D. L., Aboujaoude, E., Kuss, D. J., Pontes, H. M., Lopez Fernandez, O., Nagygyorgy, K., Achab, S., Billieux, J., Quandt, T., ... Demetrovics, Z. (2016). Working towards an international consensus on criteria for assessing internet gaming disorder: a critical commentary on Petry et al. (2014): Assessment of internet gaming disorder. Addiction (Abingdon, England), 111(1), 167-175. https://doi.org/10.1111/add.13057

Hansen, M., Armour, C., McGlinchey, E., Ross, J., Ravn, S. L., Andersen, T. E., Lindekilde, N., Elmose, M., Karsberg, S., \& Fried, E. (2021). Investigating centrality in PTSD symptoms across diagnostic systems using network analysis. European Journal of Psychotraumatology, 12(sup1), 1866412. https://doi.org/10.1080/20008198.2020.1866412

Haslam, N. (2002). Kinds of kinds: A conceptual taxonomy of psychiatric categories. Philosophy, Psychiatry, \& Psychology, 9(3), 203-217.

Jones, P. J., Mair, P., Simon, T., \& Zeileis, A. (2020). Network trees: A method for recursively partitioning covariance structures. Psychometrika, 85(4), 926-945. https://doi.org/10.1007/s11336-020-09731-4

Karhulahti, V.-M. (2020). Esport play: Anticipation, attachment, and addiction in psycholudic development. Bloomsbury Academic. 
Karhulahti, V.-M., Martončik, M., \& Adamkovič, M. (2021). Measuring internet gaming disorder and gaming disorder: A qualitative content validity analysis of validated scales. Assessment, 10731911211055436. https://doi.org/10.1177/10731911211055435

Kim, H. S., Son, G., Roh, E.-B., Ahn, W.-Y., Kim, J., Shin, S.-H., Chey, J., \& Choi, K.-H. (2022). Prevalence of gaming disorder: A meta-analysis. Addictive Behaviors, 126(107183), 107183. https://doi.org/10.1016/j.addbeh.2021.107183

King, D. L., Chamberlain, S. R., Carragher, N., Billieux, J., Stein, D., Mueller, K., Potenza, M. N., Rumpf, H. J., Saunders, J., Starcevic, V., Demetrovics, Z., Brand, M., Lee, H. K., Spada, M., Lindenberg, K., Wu, A. M. S., Lemenager, T., Pallesen, S., Achab, S., ... Delfabbro, P. H. (2020). Screening and assessment tools for gaming disorder: A comprehensive systematic review. Clinical Psychology Review, 77(101831), 101831. https://doi.org/10.1016/j.cpr.2020.101831

King, D. L., \& Delfabbro, P. H. (2014). The cognitive psychology of Internet gaming disorder. Clinical Psychology Review, 34(4), 298-308. https://doi.org/10.1016/j.cpr.2014.03.006

King, D. L., Herd, M. C. E., \& Delfabbro, P. H. (2017). Tolerance in Internet gaming disorder: A need for increasing gaming time or something else? Journal of Behavioral Addictions, 6(4), 525533. https://doi.org/10.1556/2006.6.2017.072

Kleinman, A. (1988). Rethinking psychiatry: From cultural category to personal experience. The Free Press.

Kuss, D. J., Griffiths, M. D., \& Pontes, H. M. (2017). Chaos and confusion in DSM-5 diagnosis of Internet Gaming Disorder: Issues, concerns, and recommendations for clarity in the field. Journal of Behavioral Addictions, 6(2), 103-109. https://doi.org/10.1556/2006.5.2016.062

Lemmens, J. S., Valkenburg, P. M., \& Gentile, D. A. (2015). The Internet gaming disorder scale. Psychological Assessment, 27(2), 567-582. https://doi.org/10.1037/pas0000062

Pearcy, B. T. D., Roberts, L. D., \& McEvoy, P. M. (2016). Psychometric testing of the Personal Internet Gaming Disorder Evaluation-9: A new measure designed to assess Internet Gaming Disorder. Cyberpsychology, Behavior and Social Networking, 19(5), 335-341. https://doi.org/10.1089/cyber.2015.0534

Pontes, H. M., \& Griffiths, M. D. (2015). Measuring DSM-5 internet gaming disorder: Development and validation of a short psychometric scale. Computers in Human Behavior, 45, 137-143. https://doi.org/10.1016/j.chb.2014.12.006

Pontes, H. M., Schivinski, B., Sindermann, C., Li, M., Becker, B., Zhou, M., \& Montag, C. (2019). Measurement and conceptualization of gaming disorder according to the world health organization framework: The development of the gaming disorder test. International Journal of Mental Health and Addiction. https://doi.org/10.1007/s11469-019-00088-z

Rehbein, F., Kliem, S., Baier, D., Mößle, T., \& Petry, N. M. (2015). Prevalence of Internet gaming disorder in German adolescents: diagnostic contribution of the nine DSM-5 criteria in a state-wide representative sample: Internet gaming disorder in adolescents. Addiction (Abingdon, England), 110(5), 842-851. https://doi.org/10.1111/add.12849

Sigerson, L., Li, A. Y.-L., Cheung, M. W.-L., Luk, J. W., \& Cheng, C. (2017). Psychometric properties of the Chinese Internet Gaming Disorder Scale. Addictive Behaviors, 74, 20-26. https://doi.org/10.1016/j.addbeh.2017.05.031

Smith, K. E. (2022). Disease and decision. Journal of Substance Abuse Treatment, 142(108874), 108874. https://doi.org/10.1016/j.jsat.2022.108874

Snodgrass, J. G., Zhao, W., Lacy, M. G., Zhang, S., \& Tate, R. (2019). Distinguishing core from peripheral psychiatric symptoms: Addictive and problematic Internet gaming in North 
America, Europe, and China. Culture, Medicine and Psychiatry, 43(2), 181-210. https://doi.org/10.1007/s11013-018-9608-5

Sripada, C. (2022). Impaired control in addiction involves cognitive distortions and unreliable selfcontrol, not compulsive desires and overwhelmed self-control. Behavioural Brain Research, 418(113639), 113639. https://doi.org/10.1016/j.bbr.2021.113639

Starcevic, V., Choi, T. Y., Kim, T. H., Yoo, S.-K., Bae, S., Choi, B.-S., \& Han, D. H. (2020). Internet gaming disorder and gaming disorder in the context of seeking and not seeking treatment for video-gaming. Journal of Psychiatric Research, 129, 31-39. https://doi.org/10.1016/j.jpsychires.2020.06.007

Stenseng, F., Falch-Madsen, J., \& Hygen, B. W. (2021). Are there two types of escapism? Exploring a dualistic model of escapism in digital gaming and online streaming. Psychology of Popular Media, 10(3), 319-329. https://doi.org/10.1037/ppm0000339

Van Borkulo, C., Van Bork, R., Boschloo, L., Kossakowski, J., Tio, P., Schoevers, R., Borsboom, D., \& Waldorp, L. (2017). Comparing network structures on three aspects: A permutation test. Unpublished. https://doi.org/10.13140/RG.2.2.29455.38569

van Rooij, A. J., Schoenmakers, T. M., \& van de Mheen, D. (2017). Clinical validation of the C-VAT 2.0 assessment tool for gaming disorder: A sensitivity analysis of the proposed DSM-5 criteria and the clinical characteristics of young patients with "video game addiction." Addictive Behaviors, 64, 269-274. https://doi.org/10.1016/j.addbeh.2015.10.018

World Health Organization. (2018). International classification of diseases for mortality and morbidity statistics (11th Revision). https://icd.who.int/browse11/l-m/en

Wittgenstein, L. (1953). Philosophical Investigations. Palgrave Macmillan.

Yuan, G. F., Shi, W., Elhai, J. D., Montag, C., Chang, K., Jackson, T., \& Hall, B. J. (2022). Gaming to cope: Applying network analysis to understand the relationship between posttraumatic stress symptoms and internet gaming disorder symptoms among disaster-exposed Chinese young adults. Addictive Behaviors, 124(107096), 107096.

https://doi.org/10.1016/j.addbeh.2021.107096 